staatsbürgerlichen Sinn. Wir wollen Rappaz sterben lassen, weil sein Leben weniger wert ist als unsere ethisch-medizinische Korrektheit und unsere Angst vor dem Glatteis fürsorgerischen Zwangs. Wir könnten ihn sterben lassen, weil er es ja selber will und verdient, aber dann ehrlich so und ohne Gutmenschen-Semantik. Aber wir wissen eben auch, dass es bis zuletzt eine Chance zur Umkehr gibt. Und die müssen Ärzte nutzen. Ob die Zwangsernährung möglich ist, so einfach wie es sich die Juristen vielleicht denken, ist eine berechtigte Frage. Aber es ist eine praktische Frage, die ein beherzter Arzt in Genf für sich lösen muss - unabhängig von den derzeitigen ethischen Verwirrungen.

Dr. med. Johannes G. Schmidt, Einsiedeln

\title{
Mitteilungen
}

\section{Schweizerische Gesellschaft für \\ Thorax-, Herz- und Gefässchirurgie (SGTHGC)}

\section{Preis für hervorragende} wissenschaftliche Arbeit

Die Schweizerische Gesellschaft für Thorax-, Herz- und Gefässchirurgie, bzw. ab 1.1.2011 Schweizerische Gesellschaft für Herz- und thorakale Gefässchirurgie (SGHC), verleiht jährlich eine mit 10000 Franken dotierte Auszeichnung für eine hervorragende wissenschaftliche Arbeit aus dem Gebiet der Thorax-, Herz- und Gefässchirurgie.

Die vorgelegte Arbeit darf nicht für einen anderen Preis eingereicht worden sein. Sie kann als Manuskript oder als Sonderdruck vorgelegt werden. Im Falle einer bereits erfolgten Veröffentlichung darf diese nicht länger als 6 Monate zurückliegen.

Bewerberinnen und Bewerber reichen ihre Arbeit in 4facher Ausführung bis 31.12.2010 dem Sekretär der Schweizerischen Gesellschaft für Thorax-, Herz- und Gefässchirurgie ein: Prof. Dr. med. T. Carrel, Klinik für Herzund Gefässchirurgie, Inselspital, 3010 Bern.

\section{Swiss Foundation for Tuberculosis}

\section{Research}

\section{$10^{\text {th }}$ SwissTB Award 2010}

10000 francs as Personal Prize for the best research work in the field of Tuberculosis to be granted on the World Tuberculosis Day, March 24, 2011.

General conditions:

- work accepted for publication in the year 2010;

- most of the research work done in Switzerland;

- the prize goes to the first author;

- more information at: www.swisstb.org;

- register as a SwissTBnetwork member and submit through the website.

Deadline: 31 December 2010

\section{pro integral}

\section{Eine Organisation, die Hirnverletzten} tatkräftig zur Seite steht

In der Schweiz gibt es jährlich rund 24000 Fälle schwerer Hirnverletzungen. Das Hilfswerk pro integral möchte auf diesen Sachverhalt aufmerksam machen und Betroffenen sowie ihrem Umfeld eine konkrete Hilfestellung mit Beratung, Betreuung und Begleitung bieten. Ein Schwerpunkt der Organisation ist ihr kostenloser Beratungsdienst, der rund um die Uhr zur Verfügung steht und nicht zuletzt in gewissen Aspekten für die Ärzteschaft eine Entlastung bieten könnte. Dieser Dienst steht Betroffenen mit Rat und Tat zur Seite, und es gehört zu dem Konzept von pro integral, dass die Regionalstellen von hirnverletzten Menschen geleitet werden, die den Weg zurück ins Leben wieder gefunden haben. Dies schafft Nähe zwischen den Beratern und den Betroffenen.

Einen weiteren Schwerpunkt setzt pro integral bei der Information der Bevölkerung und in die Prävention. Immerhin ist die Gefahr, eine Hirnverletzung zu erleiden, 15-mal höher als diejenige einer Querschnittslähmung. Weitere Informationen: www.prointegral.ch oder unter der kostenlosen Hotline 0800444880 